\title{
Cancer Pain at the Emergency Department and its Approach
}

\author{
B Carolina Hernández-Porras ${ }^{1 *}$, Daniela Benítez Maruri ${ }^{1}$, Silvio Namendys-Silva ${ }^{2}$ and Ricardo Plancarte \\ Sánchez ${ }^{1}$
}

${ }^{1}$ Pain Clinic, Instituto Nacional de Cancerología Mexico City, Mexico

${ }^{2}$ Intensive Care Unit, Instituto Nacional de Cancerología Mexico City, Mexico

\begin{abstract}
Introduction: Pain is reported to be a chief complaint in emergency departments (ED), however, few studies have examined pain management in the ED, and fewer in oncologic patients.
\end{abstract}

Objective: The purpose of this study was to determine the prevalence of pain complaints in the ED in cancer patients at an oncologic center.

Methods: We conducted a retrospective study in which we analyzed the consecutive, nonrandomized records of patients who arrived at the ED of the National Cancer Institute of Mexico, during the period from January 2015 to March 2015.

Results: Of 2676 patients, $30.7 \%$ complained of pain at the ED. Visceral pain was the most frequent type of pain (55.5\%), followed by somatic pain (27.4\%).

The mean VAS score of background pain was 6 (RIC 4-8), while for break through cancer pain (BTCP) the mean was 8 (RIC $7-10)$. In $92.2 \%$ of patients referred to a pain clinic office, an opioid was prescribed. Morphine, followed by tramadol and buprenorphine were the most frequent opioids prescribed.

Conclusion: This is the first study that identifies types of pain, intensity and treatment in cancer population at an ED. Recognizing pain as a prevalent problem, may be helpful to implement preventive measures or treat pain opportunely and appropriately in cancer patients. We suggest prospectively long-term studies to properly evaluate the impact of a pain clinic office in an ED at an Oncologic Center.

Keywords

Pain, Cancer pain, Emergency department

\section{Introduction}

Pain is reported to be one of the first complaints in emergency departments (ED). It is a chief complaint in up to $52.2 \%$ of visits [1].

Despite the frequency of pain in the emergency department, few studies have examined pain management in the ED. A multicenter study of an ED network documented high pain intensity and suboptimal pain management practices [2].

With regard to cancer pain, it is a prevalent symptom in the oncologic population [3-5]. Pain prevalence ranges from $33 \%$ after curative treatment, to $59 \%$ in patients on anticancer treatment and up to $64 \%$ in patients with metastatic, advanced cancer or who are in terminal phase $[6,7]$.

In 1986, the World Health Organization (WHO) proposed a strategy for cancer pain treatment based on a sequential three-step analgesic ladder from non-opioids to weak opioids to strong opioids according to pain intensity [8].
Patients should be informed about pain management and be encouraged to take an active role in their pain management. It is important to prevent the onset of pain by means of "by the clock" administration, taking into account the half-life, bioavailability and duration of action of different drugs; oral route of administration of analgesic drugs should be advocated as the first choice $[8,9]$.

Rescue dose of medications other than the regular basal therapy must be prescribed for breakthrough pain episodes

*Corresponding author: B Carolina Hernández-Porras, MD, FIPP, Pain Clinic, Instituto Nacional de Cancerología, Mexico City, San Fernando 22 Sección XVI, Tlalpan, Ciudad de México, ZC 14080, Mexico

Accepted: November 27, 2018

Published online: November 29, 2018

Citation: Hernández-Porras BC, Maruri DB, Ñamendys-Silva S, et al. (2018) Cancer Pain at the Emergency Department and its Approach. Sch J Emerg Med Crit Care 2(1):63-66 
[10]. Several studies have validated the recommendations of the analgesic ladder with good results even though originally its development was not based on evidence [11].

There are few reports about pain management in emergency departments [12]. To our knowledge, the prevalence of pain in an ED of an oncologic center has never been assessed. Valdespinoet, et al. [13] reported 10 years ago on the main symptoms and diagnoses in patients seen for consultation at the Emergency Care Service in a Mexican Comprehensive Cancer Center. They reported that the most common symptoms for emergency and urgency patient consultations were severe pain in $69.5 \%$ of cases.

On 2009, a retrospective study from Taiwan collected data about emergency visits made by cancer patients, but the setting was a general medical center [14]. However, they reported 5,488 ED visits by cancer patients, accounting for $5.6 \%$ of all ED visits made by adult patients at that center. After clustered random sampling, 1026 patients met selection criteria, and $87.9 \%$ only visited the ED once during the data collection period (one year). Pain was the chief complaint in $27.8 \%$. Other complaints were fever, shortness of breath, abdominal distention and nausea and vomiting.

\section{Objective}

The purpose of this study was to determine the prevalence of cancer pain complaints in the ED of the National Institute of Cancer in Mexico. We also report the type of painful syndrome, pain intensity and the initial treatment.

Knowing the prevalence of pain at an ED in the cancer population and identifying painful syndromes and their treatment could be essential to avoid undertreatment or inadequate management, as has been reported previously.

\section{Methods}

A retrospective study was carried out; we reviewed the consecutive, nonrandomized records of patients who presented to the ED of the National Institute of Cancer in Mexico, during the period from 1 January 2015 to 31 March 2015.

The ED has on average 44 patient visits per day. Patients can be treated by any of the following services: Surgery, Hematology and Oncology Services, after triage. The ED also includes a pain medicine office.

We collected demographic data, disease (cancer type), and the cause of the complaint. When pain was the cause of the ED visit, we recorded the presenting type of pain, according to International Association for the Study of Pain (IASP) taxonomy.

We also recorded the number of patients referred to the pain clinic office at the ED, and if it was a first or consecutive consultation. In the pain office, the taxonomic classification of pain according to the IASP taxonomy as well as back ground pain and breakthrough pain (BTCP) intensity scores using the visual analogue scale (VAS) are usually asked to patients and documented. Also, initial pharmacologic treatment was registered.
We searched in each record to ascertain whether the patient returned to the ED seven days after the first pain consultation.

Patients were excluded if data in their electronic medical record was incomplete.

\section{Data Analysis}

Descriptive statistics was performed for data submission; median and interquartile range (IR) for continuous variables with normal distribution was reported. Categorical variables were presented by frequency and percentages. The variables were analyzed with SPSS 22.0 version for Windows. Tables of contents and descriptive graphs were produced.

\section{Results}

During the first trimester of 2015, 2676 patients visited the ED. Among the 2676 patients presenting 69.6\% (1862) were women, with a median age of 54 years, IR (44-64). Demographic data and cancer type are summarized on Table 1 and Table 2, respectively. The most common cancer diagnoses in patients who visited the ED were gynecological (22\%), hematologic (16.8\%) and gastrointestinal (16.5\%). Table 1 and Figure 1 summarize demographic data and cancer types of patients presenting at the ED respectively.

Of all patients who visited the ED, 820 (30.7\%) complained of pain. Visceral pain was the most frequent type of pain described (55.5\%), followed by somatic pain (27.4\%). Somatic pain with a neuropathic component was present in $10.6 \%$ of patients, and purely neuropathic pain in $2.9 \%$. Patients who had more than one pain syndrome represented only $1.8 \%$ of the study population. See Table 2.

Of the patients who presented with pain at the ED during the period described above, 342 patients (41.7\%) required specialized pain clinic consultation.

The most common diagnoses in patients referred to the pain clinic office at the ED, were gynecological (24.3\%), breast (18.9\%) and gastrointestinal (16\%) cancer.

The mean VAS score of background pain was 6 (RIC 4-8), while for BTCP the mean was 8 (RIC 7-10). Regarding therapeutic management, it is common for physicians to

Table 1: Demographic data of cancer patients who visited the ED.

\begin{tabular}{|l|l|l|l|l|l|}
\hline \multicolumn{3}{|l|}{ Demographic data } \\
\hline Patients & Female & \multicolumn{3}{l|}{ Male } & Total \\
\hline & 1862 & $69.6 \%$ & 814 & $30.4 \%$ & 2676 \\
\hline Age & Median 54 years & \multicolumn{2}{l|}{ Interquartile range (44-64) } \\
\hline
\end{tabular}

Table 2: Different types of pain syndromes in patients at the ED.

\begin{tabular}{|l|l|l|}
\hline Pain syndromes in patients at the Ed $(\mathbf{n}=\mathbf{8 2 0})$ \\
\hline Pain syndrome & Patients $(\mathbf{n})$ & Rate \% \\
\hline Somatic & 225 & 27.4 \\
\hline Visceral & 455 & 55.5 \\
\hline Neuropathic & 24 & 2.9 \\
\hline Somatic \& secondary neuropathic & 87 & 10.6 \\
\hline Visceral \& secondary neuropathic & 14 & 1.6 \\
\hline Two pain syndromes & 15 & 1.8 \\
\hline
\end{tabular}




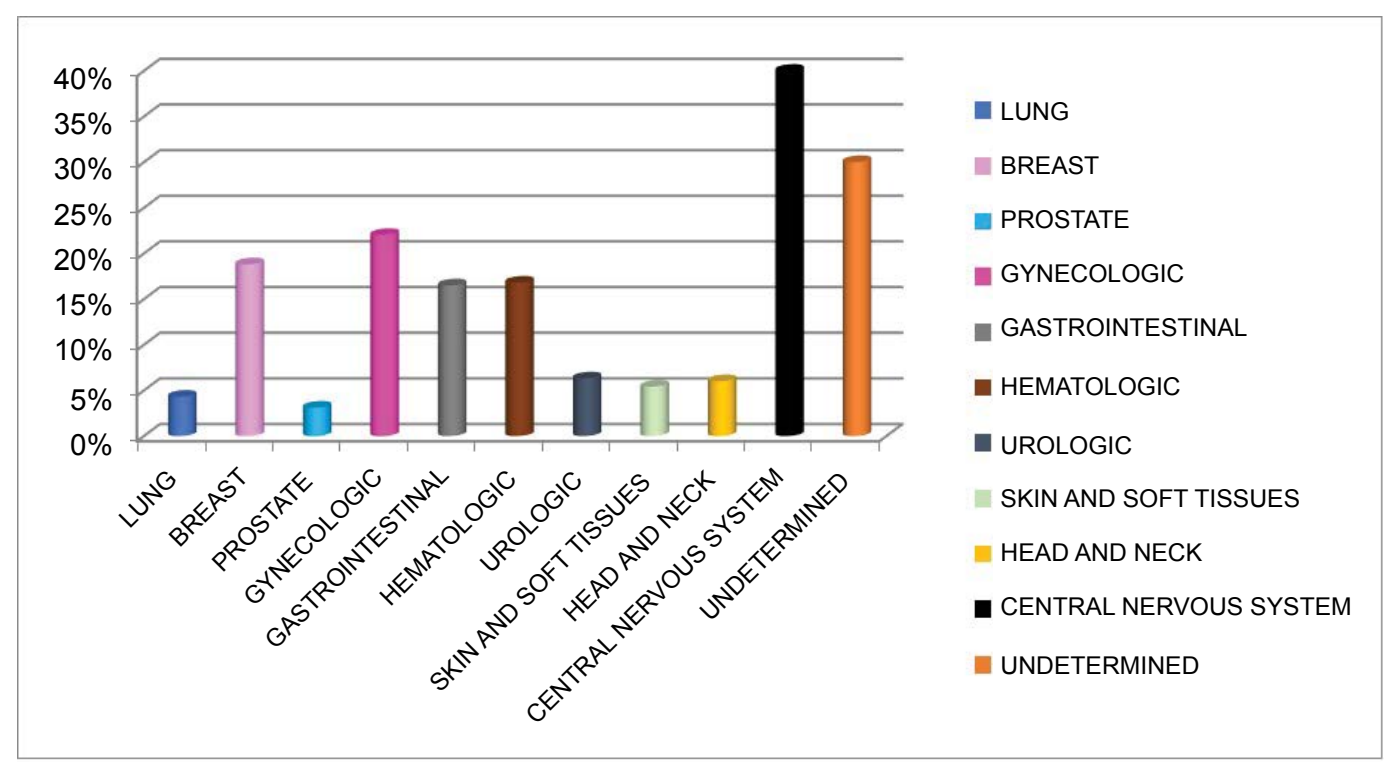

Figure 1: Cancer types of patients who visited the ED.

Table 3: Opioid Prescription at the ED.

\begin{tabular}{|c|c|c|c|c|}
\hline \multicolumn{5}{|c|}{ Opioid prescription } \\
\hline \multirow{2}{*}{\begin{tabular}{|l|} 
Opioid \\
Tramadol
\end{tabular}} & \multicolumn{2}{|c|}{$\begin{array}{l}\text { Subsequent } \\
\text { consultation (n)/(\%) }\end{array}$} & \multicolumn{2}{|c|}{$\begin{array}{l}\text { First time in pain } \\
\text { clinic }(n) /(\%)\end{array}$} \\
\hline & 118 & 34.1 & 79 & 46.7 \\
\hline Morphine & 141 & 40.8 & 52 & 30.8 \\
\hline Buprenorphine & 35 & 10.1 & 16 & 9.5 \\
\hline Fentanyl & 18 & 5.2 & 4 & 2.4 \\
\hline Oxycodone & 6 & 1.7 & 1 & 0.6 \\
\hline Not specified & 27 & 7.8 & 17 & 10.1 \\
\hline
\end{tabular}

prescribe opioids in cancer patients in order to obtain pain relief. In the deliberate search, we found that in $92.2 \%$ of patients referred to the pain clinic office, an opioid was prescribed. Morphine was the most frequent opioid prescribed, (40.8\%), followed by tramadol (34.1\%) and buprenorphine (10.1\%) as is shown in Table 3.

Specifically, in patients who consulted with the ED for first time because of pain, the more frequent opioids prescribed were tramadol (79\%), morphine (30.8\%) and buprenorphine (9.5\%).

The prescription of adjuvant analgesics occurred in $90.5 \%$ of patients. The most commonly used adjuvants were: acetaminophen (36.7\%), a gabapentinoid and acetaminophen (17.9\%) and finally, NSAIDs (15\%).

Up to $10.7 \%$ (36) of the patients returned to the ED; considering this group of patients the majority (34) (10.1\%), the main reason for consultation was pain. Of those, $9.8 \%$ required hospitalization, and it was found that only in 4 patients $(1.2 \%)$ the reason for hospitalization was secondary to intractable pain.

\section{Discussion}

Pain was a chief complaint in $27.8 \%$ of cases. Compared with the results of Valdespino, at another Mexican cancer center, pain prevalence was not as high, since they reported severe pain as an emergency and urgency patient consultation cause in $69.5 \%$ of cases. We can assume that one of the many possible reasons for this discrepancy is that the studies were conducted ten years apart, and therefore, it could be the effect of the advent and accessibility of new painkillers, radiotherapy and other drugs as adjuncts to pain relief.

A study in a Taiwan General Center, reported that among 1,179 ED visits, pain was the chief complaint in 328 visits (27.8\%). They also researched other complaints besides pain and found fever, shortness of breath, abdominal distention, and nausea/vomiting as the more frequent causes. Further analysis of pain location found that the most prevalent presenting problem was abdominal pain (54.9\%), followed by chest pain $(10.7 \%)$, whole body uneasiness/pain $(5.5 \%)$, headache (4.3\%), and oral/throat pain (4.3\%). In our study, we did not classify pain by its location, but according to the type of pain, since pain treatment can be directed based on the type and intensity of pain.

Unlike the Taiwan Center, in our Cancer Center, less than $2 \%$ of patients with pain required hospitalization because of intractable pain, while $38.8 \%$ of patients at the General Center did so. However, $10.1 \%$ of patients returned to the ED because of pain.

Of the patients with pain at the ED, $41.7 \%$ were attended at the pain clinic office, either because of the intensity of pain, or because of persistent pain following the first line of management by the clinical service. We suggest a prospectively long-term study to properly evaluate the impact of having a pain clinic in an emergency room at an Oncologic Center.

\section{Conclusion}

This is the first study that has assess pain characteristics and prevalence at an emergency department. Since the prevalence of pain at emergency rooms is high, and patients with cancer confront not only issues related to cancer 
treatment, but also to pain and to the use of analgesics, an algorithm approach must be designed to benefit patients and diminish their suffering and probably improve their quality of life.

\section{References}

1. Cordell WH, Keene KK, Giles BK, et al. (2002) The high prevalence of pain in emergency medical care. Am J Emerg Med 20: 165-169.

2. Todd KH, Ducharme J, Choiniere $M$, et al. (2010) Pain in the emergency department: Results of the pain and emergency medicine initiative (PEMI) multicenter study. J Pain 8: 460-466.

3. Nekolaichuk CL, Bruera E, Spachynski K, et al. (1999) A comparison of patient and proxy symptom assessments in advanced cancer patients. Palliat Med 13: 311-323.

4. Kutner JS, Kassner CT, Nowels DE (2001) Symptom burden at the end of life: Hospice providers' perceptions. J Pain Symptom Manage 21: 473-480.

5. Greenwald HP, Bonica JJ, Bergner M (1987) The prevalence of pain in four cancers. Cancer 60: 2563-2569.

6. Meuser T, Pietruck C, Radbruch L, et al. (2001) Symptoms during cancer pain treatment following WHO-guidelines: A longitudinal follow-up study of symptom prevalence, severity and etiology. Pain 93: 247-257.
7. van den Beuken-van Everdingen $M H$, de Rijke JM, Kessel AG, et al. (2007) Prevalence of pain in patients with cancer: A systematic review of the past 40 years. Ann Oncol 18: 1437-1449.

8. WHO (1986) Cancer pain relief. World Health Organization, Geneva.

9. Foley KM (1985) The treatment of cancer pain. N Engl J Med 313: 84-95.

10. Zech DF, Grond S, Lynch J, et al. (1995) Validation of World Health Organization guidelines for cancer pain relief: A 10-year prospective study. Pain 63: 65-76.

11. Ripamonti Cl, Santini D, Maranzano E, et al. (2012) Management of cancer pain: ESMO clinical practice guidelines. Ann Oncol 23: 39-54.

12. Tsai MC, Chen H (2002) Pain management in the emergency department. J Emerg Crit Care Med 13: 139-154.

13. Valdespino-Gómez V, López-Garza J, González-Alemán J (2006) Atención de lasemergencias y urgencias medico-quirúrgicas en un hospital oncológico. Cir Ciruj 74: 359-368.

14. Tsai SC, Liu LN, Tang ST, et al. (2010) Cancer pain as the presenting problem in emergency departments: Incidence and related factors. Support Care Cancer 18: 57-65. 